\title{
Posttraumatic Bilateral Basal Ganglia Bleed: A Report of Three Extremely Rare Cases
}

\author{
Musali Siddartha Reddy ${ }^{1} \quad$ Hemant Beniwal ${ }^{1} \quad$ Ravi Karla $^{1} \quad$ Prakash Rao Gollapudi $^{1}$ \\ ${ }^{1}$ Department of Neurosurgery, Gandhi Medical College and \\ Hospital, Padmarao Nagar, Secunderabad, Telangana, India \\ Address for correspondence Musali Siddartha Reddy, MBBS, MS, \\ (Mch), Department of Neurosurgery, 4th Floor, Gandhi Medical \\ College and Hospital, Padmarao Nagar, Secunderabad 500025, \\ Telangana, India (e-mail: siddarthareddy123@gmail.com).
}
Abstract
Keywords
- bilateral
- basal ganglia
- hematoma
- traumatic

Basal ganglia bleed, though common in hypertensive, is unusual in posttraumatic patients. Bilateral basal ganglia hematoma following trauma is extremely rare and is limited to few case reports. The authors report three cases of bilateral basal ganglia hematomas in traumatic patients. All the cases were managed conservatively.

\section{Introduction}

Traumatic basal ganglia hematoma is defined as hemorrhage in the basal ganglia or its neighboring structures such as the internal capsule or thalamus. Incidence of 3 to $10 \%$ of head injuries are reported. Bilateral basal ganglia hematoma is extremely rare and is limited to few case reports. Mechanism of basal ganglia bleed is unclear but is hypothesized to arise from sheer strain on the lenticulostriate arteries or anterior choroid vessels, caused by acceleration and deceleration forces at the time of injury. Its importance arises in the medical legal cases in which it may be taken as a nontraumatic bleed.

\section{Case Report}

Case 1

A 34-year-old man alleged to have sustained injuries in a road traffic accident (RTA) due to a fall from a two-wheeler while driving in a drunken state, was hospitalized in an unconscious state. There was history of loss of consciousness since the time of fall. There was no history of vomiting, seizures, ear/nose bleed, and cerebrospinal fluid leak following the accident. No history of a significant medical/surgical illness was reported.

On examination, the patient's Glasgow coma scale (GCS) score was E1V1M3. There was left hemiplegia. Blood pressure and pulse rate were within normal limits. The patient was intubated and put on mechanical ventilator. A computed tomography (CT) scan of the brain revealed bilateral hyperdense hemorrhagic lesions in the basal ganglia regions

(-Fig. 1). Blood parameters were within normal limits.

After stabilizing, the patient was shifted to neurosurgery intensive care unit (ICU) and put on antiepileptics, decongestants such as furosemide and mannitol $20 \%$, and antibiotics. He was continued on mechanical ventilator, and tracheostomy was done later. He deteriorated neurologically in the

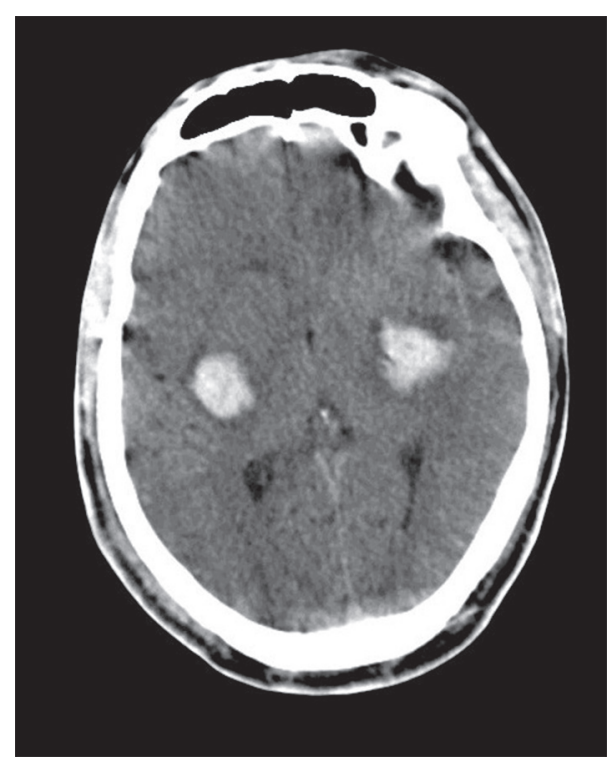

Fig. 1 Computed tomography of brain showing hyperdense lesions in bilateral basal ganglia region. received

July 6, 2018

accepted

September 6, 2018

published online

August 27, 2019
Dol https://doi.org/

10.1055/s-0039-1694850 ISSN 2277-954X.

\footnotetext{
(C2019 Neurological Surgeons' Society of India
}

License terms

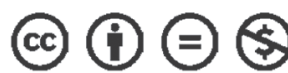


subsequent days and succumbed to cardiopulmonary arrest that was unresponsive to resuscitation.

\section{Case 2}

A 40-year-old man alleged to have sustained injuries in a RTA due to a fall from a two-wheeler under influence of alcohol presented to emergency department with complaints of loss of consciousness, vomiting, and ear bleed. The patient was nonhypertensive and nondiabetic. On examination, he was unconscious with a GCS score of E1V1M5 without any motor deficits. Pupils were mid-dilated and symmetrically reacting to light. CT scan of the brain revealed bilateral basal ganglia hematoma associated with right frontal contusion (-Fig. 2). He was intubated and put on mechanical ventilator and under the coverage of decongestants and antiepileptics. He was tracheostomized on day 4 and then weaned of the ventilator on day 7. The patient gradually regained conscious and his GCS score improved to E4V4M6. He was discharged under the coverage of antiepileptics.

\section{Case 3}

A 40-year-old woman, farmer by occupation, was brought to the emergency department by relatives with complaints of loss of conscious and vomiting following a fall from a two-wheeler as a pillion rider. On examination, the patient was drowsy with a GCS score of E3V2M5 without any motor deficits. Pupils were mid-dilated and symmetrically reacting bilaterally. She was non-hypertensive and non-diabetic. CT of the brain revealed hyperdensities in the basal ganglia region bilaterally (-Fig. 3). She was shifted to neurointensive care and was put on decongestants (mannitol) and antiepileptics (phenytoin). She gradually improved to a GCS score of E4V5M6 and was discharged after 20 days under coverage of antiepileptics.

\section{Discussion}

Hypertensive hemorrhage is common in the basal ganglia, most often restricted to one side. Traumatic basal ganglia bleed is uncommon, and its incidence among closed head injuries is reported as 3\% in few case series. ${ }^{1}$ Bilateral basal ganglia bleed is extremely rare and is restricted to few case reports. ${ }^{2,3}$ It is defined as hemorrhage in the basal ganglia and its neighboring structures such as the internal capsule and thalamus. ${ }^{4,5}$ It can be classified as large if it is greater than $2 \mathrm{~cm}$ in diameter and small if lesser than $2 \mathrm{~cm}$ in diameter.

The mechanism of traumatic basal ganglia bleed is unclear. It is most likely to be caused by shearing injury to

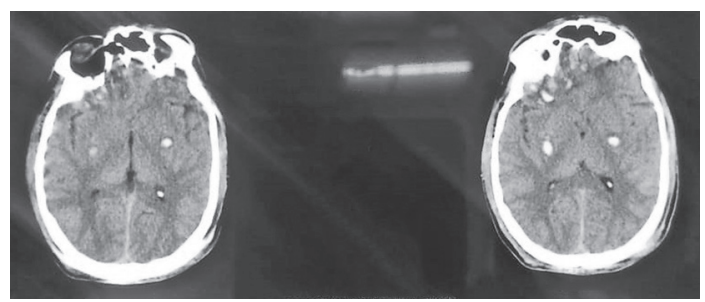

Fig. 2 Computed tomography scan of brain showing hyperdense lesions in the basal ganglia regions (left) and in the right frontal region (right).

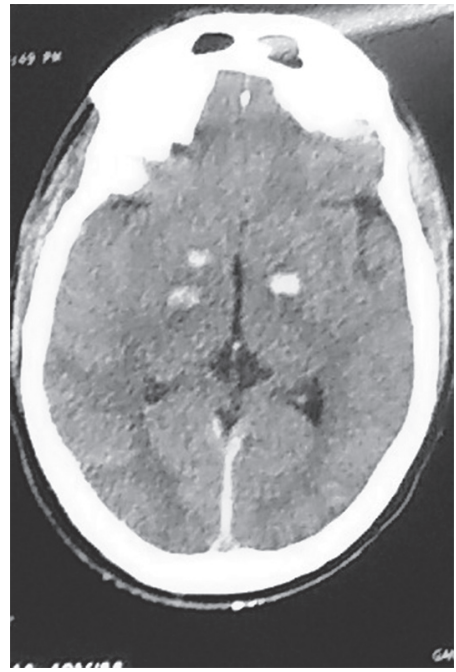

Fig. 3 Computed tomography showing two hyperdense lesions on the right and one hyperdense lesion on the left side in the basal ganglia regions.

the anterior choroidal artery and lenticulostriate artery as a result of acceleration and deceleration forces brought about by high-velocity injuries resulting in hemorrhages in basal ganglia. ${ }^{4,6}$ It has also been postulated that if trauma occurs when head is in motion, and if the impact is adequate to deform the skull is applied to the vertex, forehead, or the occipital region and directed to the tentorium, it shifts the brain through the tentorial notch producing shearing forces leading to tearing of the lenticulostriate arteries. Coup and countercoup can cause this, and this may cause bilateral lesions. $^{7}$

Here in this series, all the three cases sustained head injuries following fall from a two-wheeler, which establishes the nature of the basal ganglia bleeds. All the patients were nonhypertensive, and their blood pressures were within normal range during the length of stay, which reinstates the nature of bleeds in these cases.

Treatment options for basal ganglia hematoma include conservative, open surgery, and stereotactic guided/ultrasound-guided aspiration. ${ }^{5}$ Boto et al recommended surgical evacuation for all the lesions with a volume greater than $25 \mathrm{~mL}{ }^{5}$

Surgical evacuation was not required in any of cases in this study as intracranial pressure could be controlled under safe limit by pharmacologic means and ventilator support.

The outcomes of traumatic basal ganglia bleed have been found to be variable. The prognosis is variable and dependent on many factors such as size, associated coagulation disorders, diffuse axonal injury, age more than 60 years, and associated bleeds such as intraventricular bleeds.

Jain and colleagues reported a 38-year-old conscious man, who presented with bilateral basal ganglia hematoma and extradural hematoma. The patient underwent craniotomy and hematoma evacuation. ${ }^{8}$ Another recent report by Kaushal et $\mathrm{al}^{9}$ included a 42 -year-old male patient who suffered RTA and was hospitalized with a GCS score of 5. An isolated bilateral (mirror-image) traumatic basal ganglia bleed was revealed on CT scan of the brain. 
The patient was discharged on the 14th day. Bhargava et al reported two cases of bilateral basal ganglia bleed in 25and 50-year-old men who suffered RTA and were admitted with GCS scores of 4 each. Both were managed conservatively and discharged. ${ }^{10}$

Outcome of traumatic basal ganglia hematomas has been found to be variable. Among 37 patients studied by Boto et al, ${ }^{5}$ $59 \%$ died, $5 \%$ were vegetative, $19 \%$ experienced severe disabilities, and $16 \%$ made a favorable recovery. Katz et al, ${ }^{1}$ Jang et $a{ }^{2}{ }^{2}$ Kimura et al, ${ }^{11}$ and Lee and Wang ${ }^{12}$ have also reported good prognosis for traumatic basal ganglia hematomas. Prognosis of traumatic basal ganglia bleeds is good on comparison with other hematomas, which has been supported by all the studies.

\section{Conclusion}

Traumatic basal ganglia bleeds are rarely reported, and bilateral basal ganglia bleeds are extremely rare. It can be managed conservatively more often, and the prognosis is also variable.

\section{Conflicts of Interest}

The authors report no conflict of interest.

\section{Source of Support}

None.

\section{References}

1 Katz DI, Alexander MP, Seliger GM, Bellas DN. Traumatic basal ganglia hemorrhage: clinicopathologic features and outcome. Neurology 1989;39(7):897-904

2 Jang KJ, Jwa CS, Kim KH, Kang JK. Bilateral traumatic hemorrhage of the basal ganglia. J Korean Neurosurg Soc 2007;41:272-274
3 Yanaka K, Egashira T, Maki Y, et al. [Bilateral traumatic hemorrhage in the basal ganglia: report of two cases] [in Japanese]. No Shinkei Geka 1991;19(4):369-373

4 Adams JH, Doyle D, Graham DI, Lawrence AE, McLellan DR. Deep intracerebral (basal ganglia) haematomas in fatal non-missile head injury in man. J Neurol Neurosurg Psychiatry 1986;49(9):1039-1043

5 Boto GR, Lobato RD, Rivas JJ. Gomez PA, de la Lama A, Lagares A. Basal ganglia hematomas in severely head injured patients: clinicoradiological analysis of 37 cases. J Neurosurg 2001;94(2):224-232

6 Graham D. Neuropathology of head injury. In: Harayan PK, Wilberger JE, Povlishock JT, eds. Neurotrauma. New York, NY: McGraw-Hill; 1996:43-59

7 Fujioka M, Maeda Y, Okuchi K, Kagoshima T, Taoka T. Secondary change in the substantia nigra induced by incomplete infarct and minor hemorrhage in the basal ganglia due to traumatic middle cerebral arterial dissection. Stroke 1999;30(9):1975-1977

8 Jain SK, Sundar IV, Sharma V, Prasanna KL, Kulwal G, Tiwari RN. Bilateral large traumatic basal ganglia haemorrhage in a conscious adult: a rare case report. Brain Inj 2013;27(4):500-503

9 Kaushal R, Kataria R, Gupta A, Sinha V. Traumatic bilateral (mirror-image) basal ganglia bleed. The Indian Journal of Neurotrauma. 2011;8(2):109-110

10 Bhargava P, Grewal SS, Gupta B, Jain V, Sobti H. Traumatic bilateral basal ganglia hematoma: a report of two cases. Asian. J Neurosurg 2012;7(3):147-150

11 Kimura M, Sobata E, Suzuki S, Nonogaki Y, Iwabuchi T. [Traumatic hematoma in the basal ganglia (caudate) with favorable prognosis: report of two cases] [in Japanese]. No Shinkei Geka 1994;22(2):155-158

12 Lee JP, Wang ADJ. Post-traumatic basal ganglia hemorrhage: analysis of 52 patients with emphasis on the final outcome. J Trauma 1991;31(3):376-380 